\title{
Reversal of TET-mediated 5-hmC loss in hypoxic fibroblasts by ascorbic acid
}

\author{
Yukun Liu ${ }^{1,2,3} \cdot$ Shuyun $\mathrm{Xu}^{1} \cdot$ Tingjian $\mathrm{Zu}^{1} \cdot$ Feng $\mathrm{Li}^{1} \cdot$ Shengbo Sang ${ }^{1} \cdot$ Cynthia Liu ${ }^{1} \cdot \mathrm{Yang} \mathrm{An}^{2} \cdot{\text { Bobin } \mathrm{Mi}^{2}}^{2}$ \\ Dennis P. Orgill ${ }^{2}$ - George F. Murphy ${ }^{1}$. Christine G. Lian ${ }^{1}$
}

Received: 30 August 2018 / Revised: 16 December 2018 / Accepted: 30 December 2018 / Published online: 5 March 2019

(c) United States \& Canadian Academy of Pathology 2019

\begin{abstract}
Hypoxia resulting in hypoxia-inducible factor-1 alpha (HIF-1 $\alpha$ ) induction is known to drive scar formation during cutaneous wound healing, and may be responsible for excessive fibrosis inherent to hypertrophic scars and keloids. Because epigenetic pathways play an important role in regulation of fibrosing processes, we evaluated patient scars for DNA hydroxymethylation (5-hydroxymethylcytosine; 5-hmC) status and documented a significant decrease in scar fibroblasts. To test this finding in vitro, human fibroblasts were cultured with cobalt chloride $\left(\mathrm{CoCl}_{2}\right)$, a known stimulant of HIF-1 $\alpha$. HIF-1 $\alpha$ induced so resulted in loss of 5-hmC similar to that seen in naturally occurring scars and was associated with significant downregulation of one of the 5-hmC converting enzymes-ten-eleven translocation 3 (TET3) - as well as increased expression of phosphorylated focal adhesion kinase (p-FAK), which is important in wound contracture. These changes were partially reversed by exposure to ascorbic acid, a recognized epigenetic regulator potentially capable of minimizing excessive scar formation and promoting a more regenerative healing response. Our results provide a novel and translationally relevant mechanism whereby epigenetic regulation of scar formation may be manipulated at the level of fibroblast DNA hydroxymethylation.
\end{abstract}

\section{Introduction}

Wounds heal postnatally by forming scars in which differentiation programs are restricted to the formation of myofibroblasts that collaborate to form a contractile syncytium, and ultimately the genesis of collagen-secreting fibroblasts $[1,2]$. The result is a non-regenerative patch that does not express many of the original functions of the damaged

George F. Murphy

gmurphy@bwh.harvard.edu

$\bowtie$ Christine G. Lian

cglian@bwh.harvard.edu

1 Program in Dermatopathology, Department of Pathology, Brigham and Women's Hospital, Harvard Medical School, Boston, MA, USA

2 Division of Plastic Surgery, Department of Surgery, Brigham and Women's Hospital, Harvard Medical School, Boston, MA, USA

3 Department Plastic Surgery, Tongji Hospital, Tongji Medical College, Huazhong University of Science and Technology, Wuhan 430030, China tissue and that may be cosmetically unattractive, particularly in the setting of hypertrophic scar and keloid formation [3]. Although knowledge of the precise mechanisms responsible for scar formation remain as yet incomplete, hypoxia has been shown to play an important role in the generation of hypertrophic scars [4]. Moreover, the partial pressure of oxygen $(\mathrm{pO} 2)$ is significantly decreased in scar tissue in comparison to normal human dermis [5]. Hypoxiainducible factor-1 alpha (HIF1 $\alpha)$ is an important determinant of cellular responses to oxygen deprivation [6,7], and HIF1 $\alpha$ protein levels are known to be elevated in keloid tissue due to oxygen deprivation $[8,9]$. The potential role of hypoxia in the induction of the differentiation-restricted myofibroblast/fibroblast that typifies scar formation has not as yet been fully explored, particularly in the context of genetic and epigenetic regulators of cellular plasticity that would be required for non-cicatricial regenerative healing responses [10].

Globally, scar formation is regulated by genetic and epigenetic influences. Epigenetic regulation involves three main mechnisms: DNA methylation/hydroxymethylation, histone modification, and non-coding RNAs [11]. Epigenetic modifications refer to potentially heritable alterations 
without changes in DNA sequence [12]; they are often reversible and therefore subject to experimental and therapeutic manipulation. DNA methylation/hydroxymethylation plays a vital role in maintaining normal cell types and tissue functions. In the process of DNA methylation, DNA methyltransferases (DNMTs) first form 5-methylcytosine from cytosine, with subsequent demethylation of 5methylcytosine to 5-hydroxymethylcytosine $(5-\mathrm{hmC})$ by the ten-eleven translocation (TET) family of enzymes (TET1-3) through addition of a hydroxyl group. 5-hmC, which is found in a variety of normal tissues and cell types, is an important epigenetic marker known to govern fibroblast plasticity [13]. Previous studies have implicated DNA methylation events in the differentiation of keloidproducing myofibroblasts and fibroblasts [14, 15]. Furthermore, DNA methylation is involved in fibrosing disorders of the liver, lungs, and heart [16]. Of potential relevance, loss of 5-hmC has been found in melanoma as well as in many other cancer cell types [17, 18], all processes involving cell proliferation in response to hypoxia [19]. In forming scar tissue, tumor-related genes may also be mutated or overexpressed in fibroblasts [20-22]. Accordingly, this study was undertaken to test the hypothesis that abnormalities in DNA hydroxymethylation may also characterize the formation of cutaneous scars.

\section{Materials and methods}

\section{Immunohistochemical staining of human scar tissue}

This study was conducted with approval of the Institutional Review Board of Brigham and Women's Hospital, Harvard Medical School. Deidentified scar tissue specimens $(n=10$; including hypertrophic scars), and normal skin control specimens $(n=10)$ were retrieved from the archives of the Department of Pathology at Brigham and Women's Hospital. Hematoxylin and eosin (H\&E) staining was performed for histological evaluation. For 5-hmC immunohistochemical (IHC) staining, tissue sections were deparaffinized and blocked according to a established protocols [17]. Antigen retrieval was accomplished with DAKO Target Antigen Retrieval solution in a pressure cooker, as previously published [17]. Slides were incubated overnight at $4{ }^{\circ} \mathrm{C}$ with primary anti-5-hmC antibody (1:1500, Cat No. 39769, Active Motif, Carlsbad, CA). The slides were then incubated for $1 \mathrm{~h}$ at room temperature with peroxidase-conjugated secondary goat anti-rabbit $\operatorname{IgG}$ (1:200, Cell signaling, Danvers, MA) followed by development of chromogenic substrate for $3 \mathrm{~min}$ and counterstained with hematoxylin. Slides were mounted with Permount ${ }^{\mathrm{TM}}$, covered with a coverslip, and observed under a microscope. Evaluation of 5-hmC staining was analyzed using the following scoring system for evaluation of dermal spindle cells: 0 (no staining), $1+$ (weak and incomplete nuclear staining), $2+$ (strong, complete nuclear staining in $<30 \%$ of cells or weak/moderate heterogeneous nuclear staining in more than $10 \%$ of cells), and $3+$ (strong complete homogenous nuclear staining in $30 \%$ or more of cells) [23]. Counting and scoring were done by three pathologists blinded who were previously trained in the HER2 scoring system.

\section{Cell culture}

Human dermal fibroblast cells were purchased from ScienCell (Cat No. 2320, ScienCell, Carlsbad, CA). DMEM-Dulbecco's Modified Eagle Medium supplemented with $10 \%$ fetal bovine serum (FBS), $50 \mathrm{U} / \mathrm{ml}$ penicillin, and $50 \mathrm{mg} / \mathrm{ml}$ streptomycin (Cat No. 15140-122, Thermo Fisher Scientific, Waltham, MA) was used as a complete media. Cells were cultured in an incubator with $5 \% \mathrm{CO}_{2}$ at $37^{\circ} \mathrm{C}$.

\section{Hypoxia and ascorbic acid treatment}

To mimic hypoxic conditions, the fibroblasts were treated with cobalt chloride $\left(\mathrm{CoCl}_{2}\right)$ (Cat No. C8661-25G, SigmaAldrich, St. Louis, MO) in different concentrations at 100 and $200 \mu \mathrm{M}$ for $24 \mathrm{~h}$. The cells were then treated with ascorbic acid (AA) (Cat. No. BP351-500, Thermo Fisher Scientific Waltham, MA) for another $24 \mathrm{~h}$ at $100 \mu \mathrm{M}$. There were two control groups: cells which received no treatment as a negative control and cells treated only with ascorbic acid for $24 \mathrm{~h}$. Treatments were completed in the incubator with $5 \% \mathrm{CO}_{2}$ at $37^{\circ} \mathrm{C}$, and experiments were repeated three times.

\section{Western blot analysis}

Cells were collected and lysed with $100 \mu \mathrm{l}$ lysis buffer (100 mM Tris PH 6.8, 2\% SDS and 12\% glycerinum) on ice. The cell lysates were then sonicated and centrifuged at $12,000 \mathrm{rpm}$ for $25 \mathrm{~min}$. A BCA kit (Thermo Scientific, Waltham, MA) was used to collect supernatants and detect protein concentration. Cell lysates were added with loading buffer and heated at $100{ }^{\circ} \mathrm{C}$ for $5 \mathrm{~min}$, and centrifuged to obtain protein which was then electrophoresed in running buffer on Mini-protean ${ }^{\circledR}$ TGX $^{\mathrm{TM}}$ Precast Gel (Bio-Rad, Hercules, CA) and transferred to Nitrocellulose membranes in transfer buffer. Membranes were blocked in PBS containing 5\% non-fat dry milk and $0.1 \%$ Tween 20 at room temperature for $1 \mathrm{~h}$. Primary antibodies-HIF-1 $\alpha$ (1:1000, Cat No. 458400, Invitrogen, Carlsbad, CA), TET3 (1:1000, Cat No. GTX121453, Gene Tex, Irvine, CA), p-FAK and FAK (1:1000, Cat No. 8556P, Cat No. 3285P, Cell signaling, Danvers, MA)—were immunoblotted at $4{ }^{\circ} \mathrm{C}$ 
overnight on a shaker. After washing with PBS Tween20 for $5 \mathrm{~min}$ and repeating three times, the membrane was then incubated with goat anti-mouse and goat anti-rabbit IgGHRP (1:2000, Cat No. 7076V, 7074V, Cell Signaling, Danvers, MA) separately for $1 \mathrm{~h}$ at room temperature. Exposure of protein band and densitometry measurement were completed by ChemiDOC ${ }^{\mathrm{TM}} \mathrm{XRS}^{+}$with Image Lab system (Bio-Rad, Hercules, CA).

\section{Real-time polymerase chain reaction}

After treatment with $\mathrm{CoCl}_{2}$ and ascorbic acid, experimental and control cells were collected for total RNA extraction using a RNeasy ${ }^{\circledast}$ mini kit (Cat No. 74104, Qiagen, Hilden, Germany) according to the manufacturer's protocol. The RNA concentration was measured using a NanoDrop spectrophotometer (Thermo Fisher Scientific, Waltham, MA). Purified RNA from fibroblasts was reverse transcribed by adding RNA sample $1 \mu \mathrm{g}, 5 \times$ reverse transcriptase reaction mix (Cat No. 1708840, Bio-Rad, Hercules, CA) with RNase-free water to constitute the $20 \mu \mathrm{l}$ cDNA synthesis reaction. T100 ${ }^{\mathrm{TM}}$ PCR system (BioRad, Hercules, CA) was used for cDNA synthesis according to protocol. Then the cDNA reaction products were mixed with forward and reverse primers. The primers used for target genes were: human TET3 forward: 5'-CGATTGCGTCGAACAA ATAG-3' and reverse: 5'-CTCCTTCCCCGTGTAGATGA-3'; human PTk2(FAK) forward: 5'-GCGCTGGCTGGAAAAAG AGGAA-3' and reverse: 5'-TCGGTGGGTGCTGGCTG GTAGG-3'; housekeeping gene GAPDH forward: 5'-CT CCTCCTGTTCGACAGTCAGC-3' and reverse: $5^{\prime}$-CCATGGAATTTGCCATGGGTGG-3'; and housekeeping gene 18s forward: 5'-GTAACCCGTTGAACCCCATT-3' and reverse: 5'-GGCCTCACTAAACCATCCAA-3'. For each sample, $1 \mu \mathrm{l}$ cDNA was mixed with $10 \mu$ l Power $\mathrm{SYBR}^{\circledR}$ Green Master Mix (Cat No.172-5121, Bio-Rad, Hercules, CA) and $8 \mu$ RNasefree water containing $1 \mu \mathrm{l}$ primers for a total volume of $20 \mu \mathrm{l}$. Semiquantitative real-time polymerase chain reaction (qPCR) was performed by CFX96 real-time PCR detection system (Bio-Rad, Hercules, CA) with Bio-Rad CFX manager software (Bio-Rad, Hercules, CA). All samples for qPCR reactions were run in triplicate. Fold-change in RNA levels was calculated using the $2^{-\Delta \Delta \mathrm{Ct}}$ method.

\section{5-hmC dot blot and capture}

DNA was extracted by QIAamp ${ }^{\circledR}$ DNA Mini Kit Cells pellet according to the protocol provided. DNA concentration was determined by a NanoDrop spectrophotometer (Thermo Fisher, Waltham, MA). After sonicating DNA into $\sim 200 \mathrm{bp}$ size on a Mosonix Sonicator (LabX), DNA samples were diluted to $50 \mathrm{ng} / \mu \mathrm{l}$ using a DE buffer to three decreasing concentrations of 200,100 and $50 \mathrm{ng} / \mu \mathrm{l}$, respectively. Samples were placed in a 96-well plate with $20 \mu \mathrm{l} \mathrm{M}$
$\mathrm{NaOH} / 25 \mathrm{mM}$ EDTA per well at $95^{\circ} \mathrm{C}$ for $10 \mathrm{~min}$ by BioRad Thermal cycle and put on ice immediately. Then $50 \mu \mathrm{l}$ $2 \mathrm{M}$ iced ammonium acetate was added to each well. A BioDot SF Microfiltration Apparatus (Bio-Rad, Hercules, CA) was set up with nitrocellulose membranes and connected to negative pressure. Denatured DNA was added to the wells and DNA was fixed to the membrane by UV 2400 for 10 min. The membrane was blocked with $5 \%$ non-fat milk for $1 \mathrm{~h}$ and incubated overnight with anti-5-hmC antibody (1:2000, Cat No.39769, Active Motif, Carlsbad, CA). The membrane was then incubated for $1 \mathrm{~h}$ with goat anti-mouse IgG-HRP (1:2000, SC-2031, Cruz Marker, Dallas, TX). The membrane was hybridized with $0.02 \%$ methylene blue for 10 min to stain DNA. To determine the DNA expression of 5-hmC, band exposure and densitometry measurement were completed by ChemiDOC ${ }^{\mathrm{TM}} \mathrm{XRS}^{+}$and Image Lab system (Bio-Rad, Hercules, CA).

\section{Immunofluorescence staining}

For immunocytochemistry, cells were fixed in $100 \%$ acetone for $15 \mathrm{~min}$ and washed with PBS-Tween 20 three times. Cells were permeabilized with $0.5 \%$ Triton X-100 in PBS for $15 \mathrm{~min}$. For 5-hmC staining, sections were incubated in $4 \mathrm{~N} \mathrm{HCl}$ at room temperature for $30 \mathrm{~min}$, then neutralized with $100 \mathrm{mM}$ Tris- $\mathrm{HCl}(\mathrm{pH} 8.5)$ for $15 \mathrm{~min}$, and finally followed by $1 \%$ BSA blocking at room temperature for $1 \mathrm{~h}$. For p-FAK and F-actin staining, sections were blocked with $1 \%$ BSA after being permeabilized. Then cells were incubated with primary antibodies for $2 \mathrm{~h}$ at $37^{\circ} \mathrm{C}$, and secondary antibodies for $1 \mathrm{~h}$ at room temperature. Primary antibodies were used as follows: anti-5-hmC (1:1500, Cat No. 39769, Active Motif, Carlsbad, CA), p-FAK (1:100, Cat No. 3285P, Cell signaling, Danvers, MA), F-actin (1:100, Cat No. Ab130935, Abcam, Cambridge, UK). Secondary antibodies were anti-rabbit Alexa Fluor 594 (1:2000, Cat No. A11037, Invitrogen, Carlsbad, CA) and anti-mouse IgM FITC (1:2000, Cat No. 553437, Bioscience, Billerica, MA). After incubation with antibodies, sections were washed and stained with DAPI (Cat No. P36935, Invitrogen, Carlsbad, CA). Slides were observed by immunofluorescence microscopy and images were captured by Cytovision software (Wetzlar, Germany).

\section{Statistical analysis}

To compare differences between groups with different treatments. One-way ANOVA analysis of variance followed by Bonferroni's multiple comparisons test was performed using GraphPad Prism software (GraphPad Prism version 6.00, GraphPad Software, USA). Statistical significance was set as $p$ value $<0.05$. All data were presented as means \pm standard deviation (SD). 

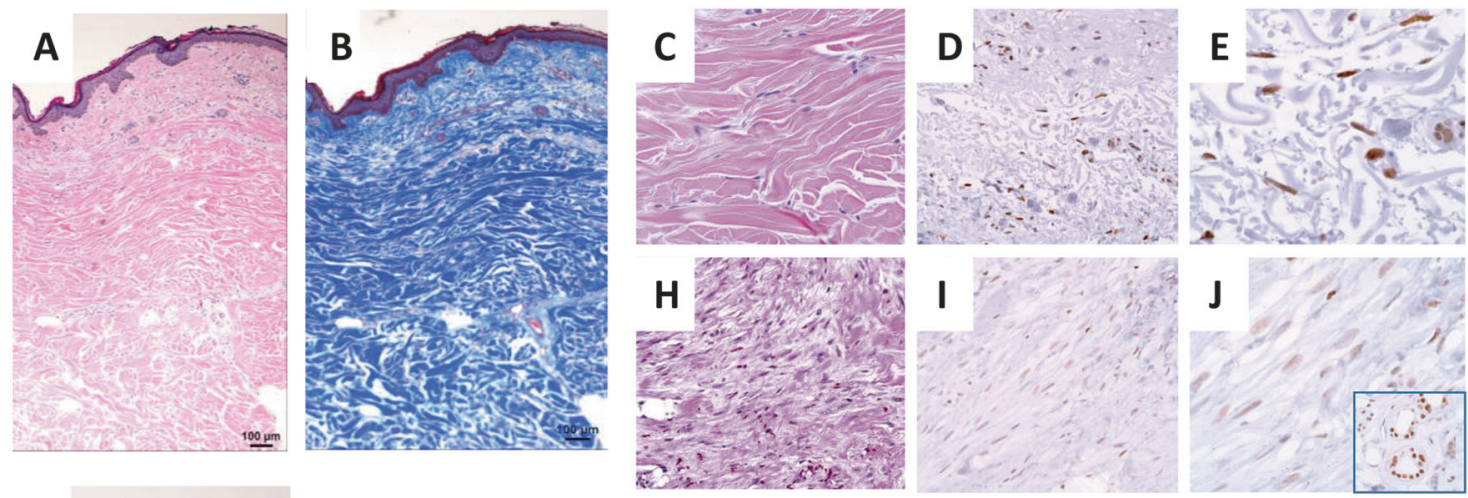
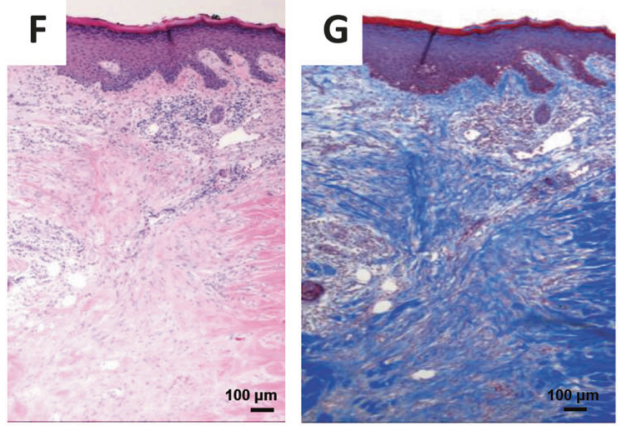

Fig. 1 Immunohistochemical (IHC) staining for 5 -hmC in clinical samples. a, c Normal skin stained by H\&E and b trichrome revealing interlacing collagen bundles; f, h $\mathrm{H} \& \mathrm{E}$ and $\mathbf{g}$ trichrome-stained hypertrophic scar showing hypercellular dermis with randomly-

\section{Results}

\section{Human scar fibroblasts show loss of 5-hmC}

In contrast to normal control skin (Fig. 1a-c), patient scars (both conventional surgical scars and hypertrophic scars; Fig. 1f-j) showed variable epidermal architecture, hypercellular dermis with increase in compacted collagen bundles (highlighted by trichrome staining; Fig. 1b, g), and significantly less 5-hmC nuclear immunoreactivity in dermal fibroblasts than in randomly oriented fibroblasts in the normal human dermis (Fig. 1i, j vs d, e). An immunohistochemistry scoring system was used for 5-hmC expression evaluation (Fig. 1k). These findings suggested a role for loss of DNA hydroxymethylation in cutaneous scar formation, a process known to be associated with fibroblast hypoxia.

\section{Effect of $\mathrm{CoCl}_{2}$ and ascorbic acid on fibroblast cytomorphology and HIF-1a expression}

Exposure of cultured human dermal fibroblasts to $\mathrm{CoCl}_{2}$ resulted in changes in subtle cell morphology that consisted of a decrease in culture density and more variability in cell contour to include less spindled, plumper cytomorphology (Fig. 2a). HIF-1 $\alpha$ induction by $\mathrm{CoCl}_{2}$ was confirmed by western blotting (Fig. 2b, c). Addition of ascorbic acid to cultures resulted in partial reversal of these

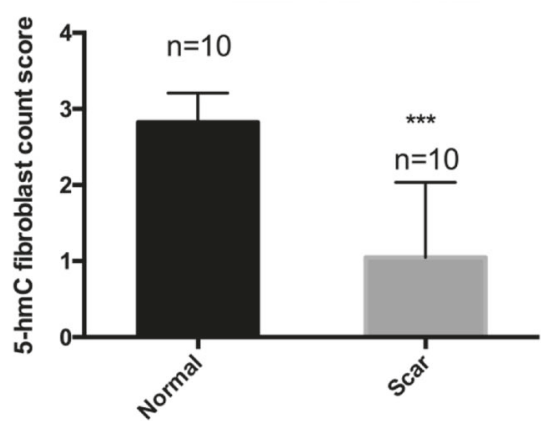

oriented, tightly packed collagen bundles. d, e Normal skin fibroblasts by IHC with strong nuclear 5 -hmC reactivity compared to $\mathbf{i}, \mathbf{j}$ fibroblasts in hypertrophic scars (inset in $\mathbf{j}=$ positive internal control eccrine gland; $\mathbf{k}=$ quantitative comparison)

cytomorphologic alterations and a decrease in HIF- $1 \alpha$ expression (Fig. 2a-c). These findings supported the specificity of the effect of $\mathrm{CoCl}_{2}$ in the induction of hypoxia, and suggested that related cytomorphologic alterations may affect cell adhesion or cytoskeletal integrity. Further evaluation using Western blotting of effects of $\mathrm{CoCl}_{2}$ and ascorbic acid on the TET enzyme family revealed a decrease in TET3 protein expression as a result of $\mathrm{CoCl}_{2}$ treatment, with partial to complete reversal of these effects by ascorbic acid (Fig. 2d, e), further suggesting a role for the TET/5-hmC axis in scar formation.

\section{$\mathrm{CoCl}_{2}$ and ascorbic acid alter fibroblast 5-hmC and TET3 expression}

By immunofluorescence staining and dot-blot, fibroblast 5 -hmC was significantly reduced in the 100 and $200 \mu \mathrm{M}$ $\mathrm{CoCl}_{2}$ concentration range (Fig. $3 \mathrm{a}-\mathrm{c}$ ), and these changes were reversed by ascorbic acid. To further explore the possible role of TET family enzymes in hypoxia-induced 5-hmC loss, we examined TET transcriptional status using qRT-PCR. TET3 mRNA, but not TET1 or TET2 (data not shown), was diminished in association with $\mathrm{CoCl}_{2}$-induced HIF-1 $\alpha$, and this effect was also reversed by ascorbic acid (Fig. 3d). These findings supported TET protein expression data as obtained by western blotting (see Fig. 2d, e). 

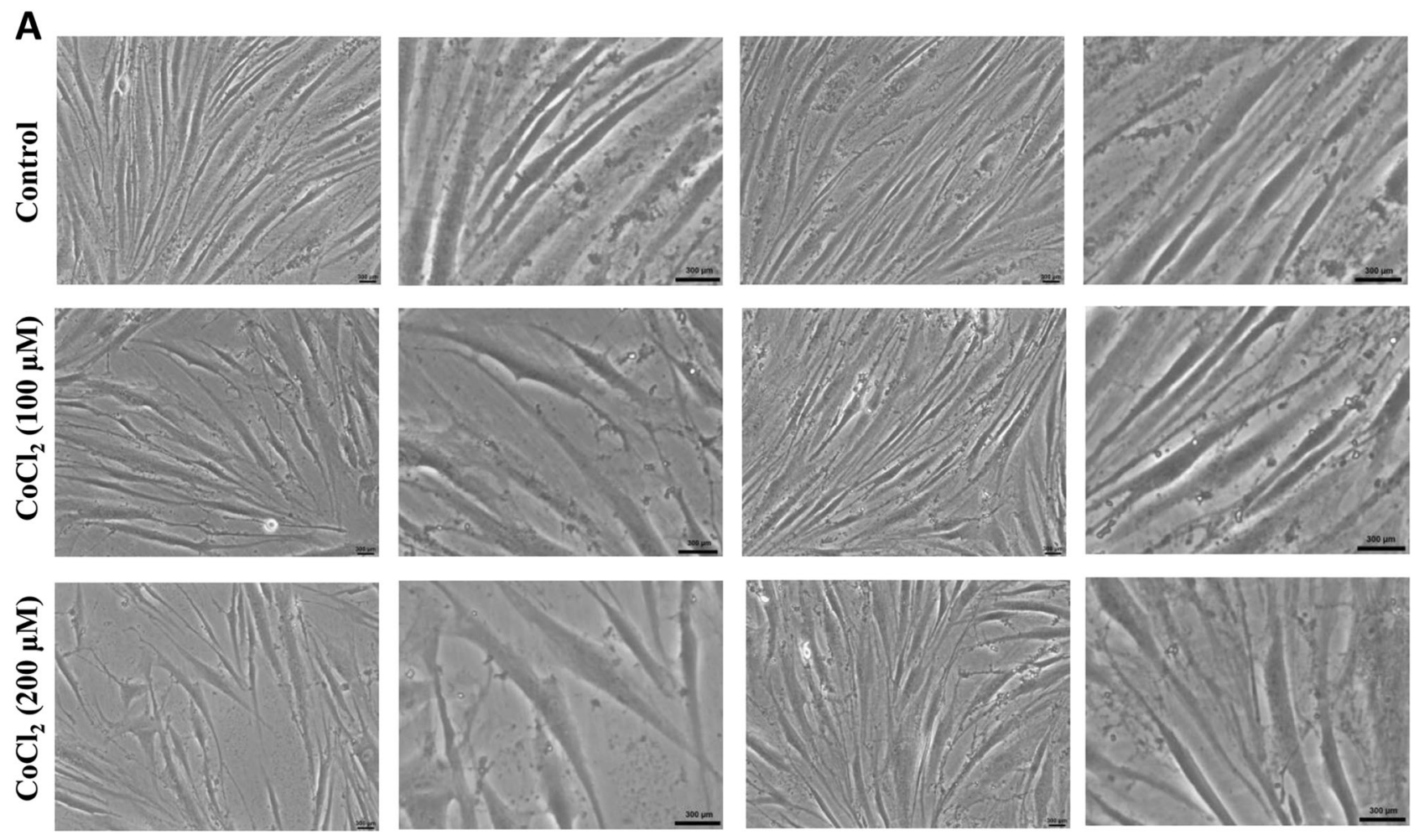

\section{No AA treatment control}

\section{AA $(100 \mu M)$ for 24 hours}

B
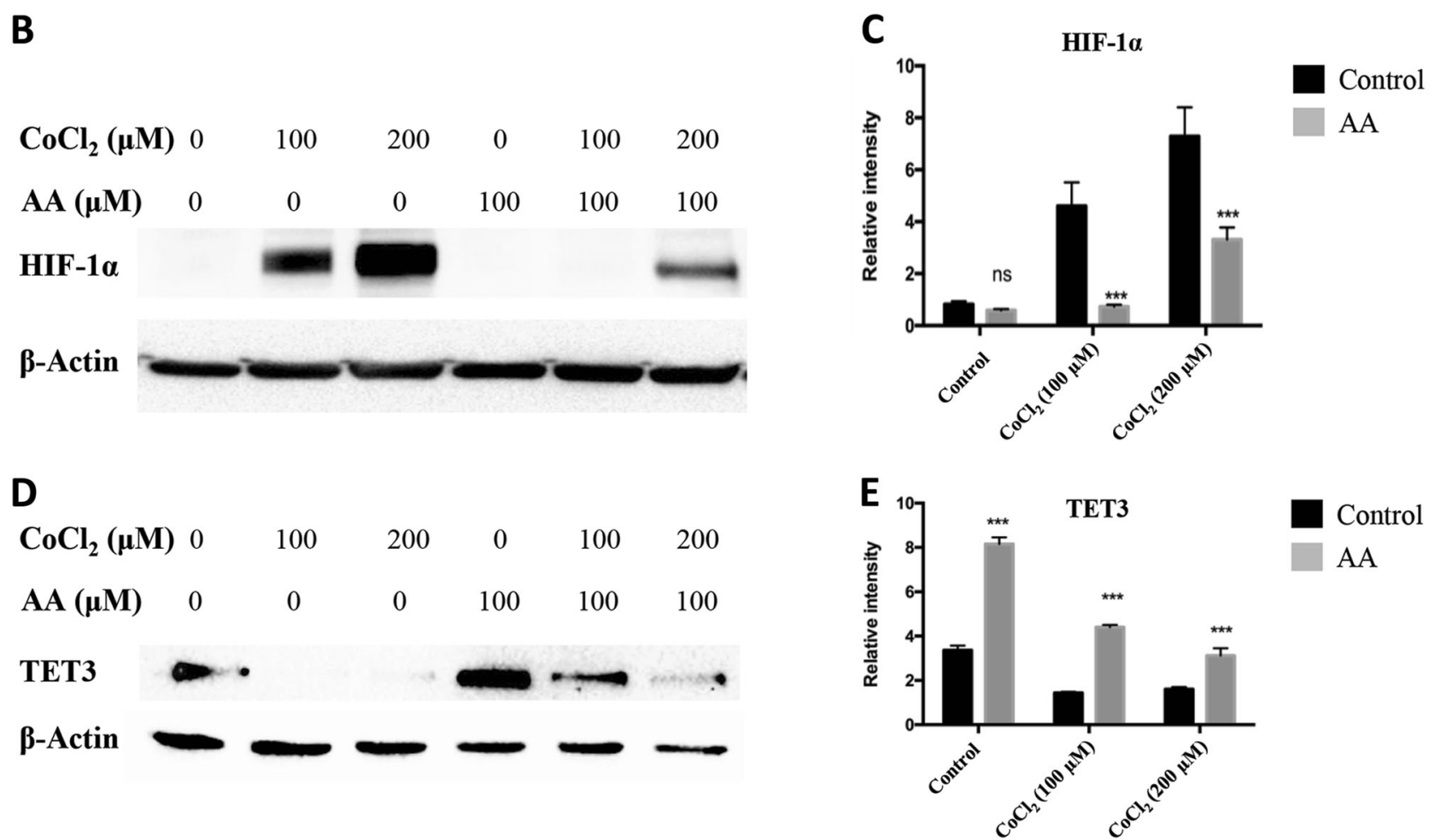

Fig. 2 Cultured human fibroblasts exposed to $\mathrm{CoCl}_{2}$. a Phase contrast of fibroblasts exposed to 100 and $200 \mu \mathrm{M}$ of $\mathrm{CoCl}_{2}$; subtle alterations in cytomorphology consist of less cellular monolayers formed by slightly plumper, less spindled cells. b, c Western blots show increased

HIF- $1 \alpha$ and d, e decreased TET3 protein expression as a result of $\mathrm{CoCl}_{2}$ treatment, and partial to complete reversal of these effects by ascorbic acid (AA). $(* * * p<0.05 ; \mathrm{ns}=$ no significance) 

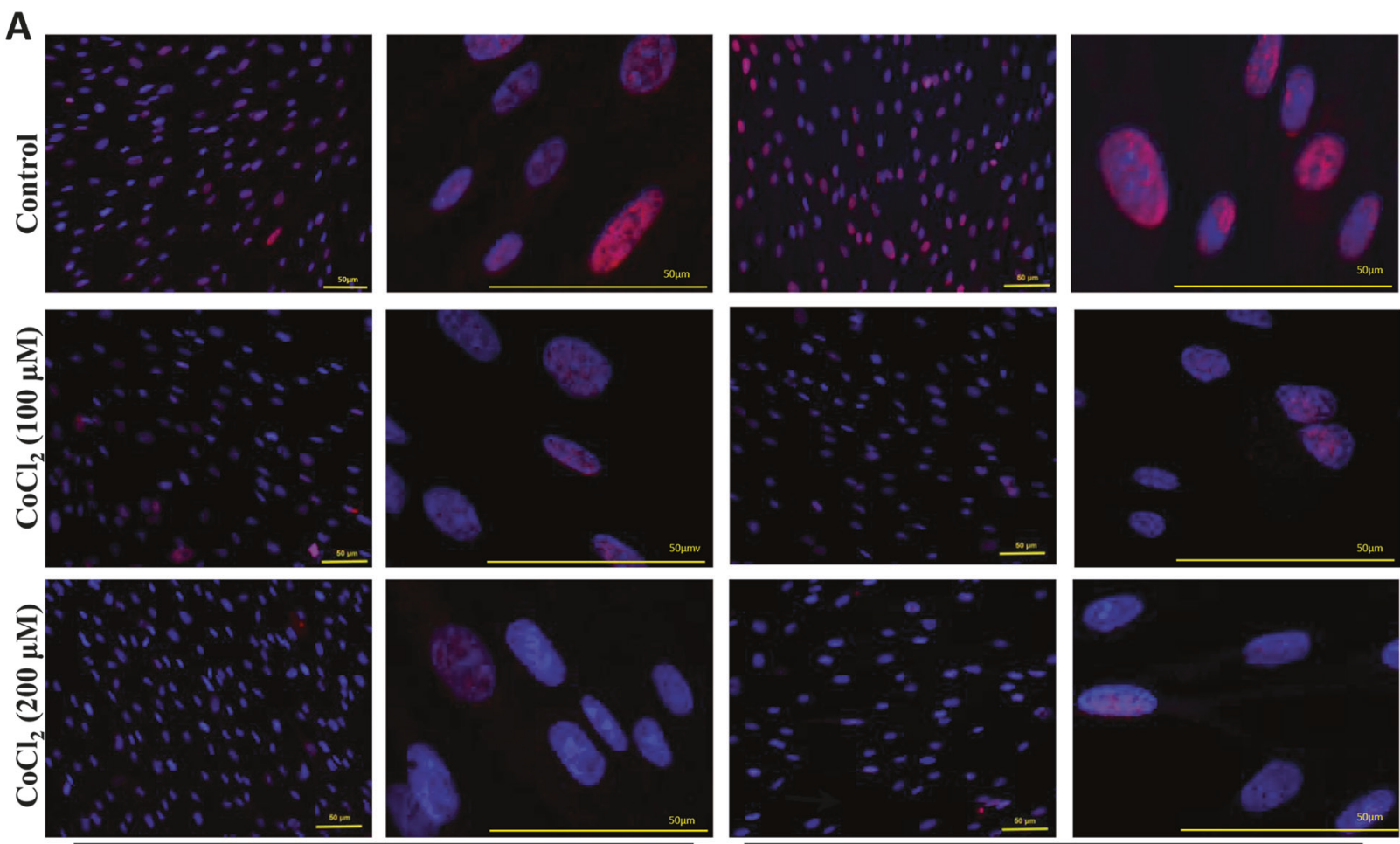

No AA treatment control

AA $(100 \mu \mathrm{M})$ for 24 hours

B

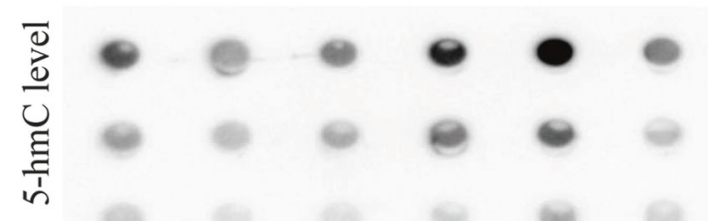

$1.0 \mu \mathrm{g}$

$0.5 \mu \mathrm{g}$

$0.25 \mu \mathrm{g}$

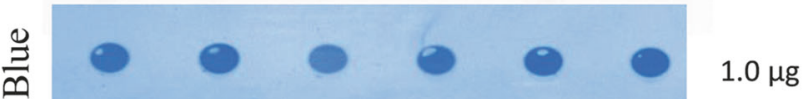

C

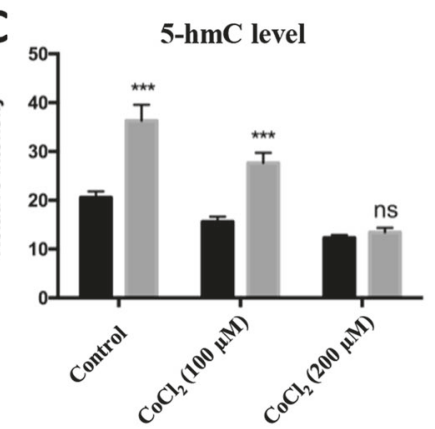

$0.5 \mu \mathrm{g}$

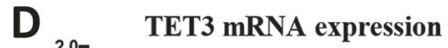

$0.25 \mu \mathrm{g}$

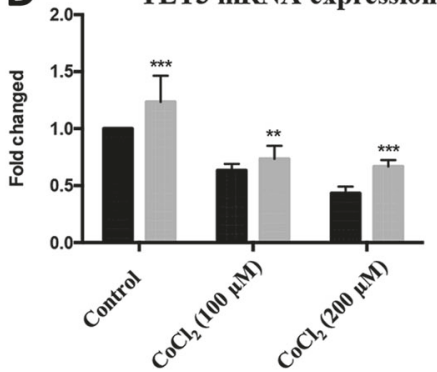

Control

AA

Control

AA

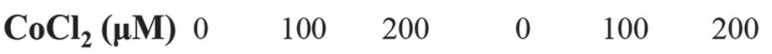

$\begin{array}{lllllll}\mathbf{A A}(\boldsymbol{\mu M}) & 0 & 0 & 0 & 100 & 100 & 100\end{array}$

Fig. 3 Effects of $\mathrm{CoCl}_{2}$ and AA treatment of human fibroblasts on 5hmC. a Immunofluorescence (IF) staining (red -5 -hmC, blue $=$ DAPI) and b, c dot blot, showing $\mathrm{CoCl}_{2}$-induced decrease in 5-hmC

\section{Ascorbic acid inhibits p-FAK expression in hypoxic fibroblasts}

To investigate the possible involvement of FAK/phosphorylated FAK (p-FAK) in this pathway, we used qRT-

DNA is restored by AA treatment that also results in $\mathbf{d}$ upregulation of TET3 mRNA by qRT-PCR. $(* * p<0.05 ; * * * p<0.001 ; \mathrm{ns}=$ no significance)

PCR and Western blotting to confirm increased expression in association with induction of fibroblast hypoxia. We found increased p-FAK mRNA and protein under hypoxic conditions, which was further confirmed by dual immunofluorescence staining for F-actin and p-FAK (Fig. 4a-d). 


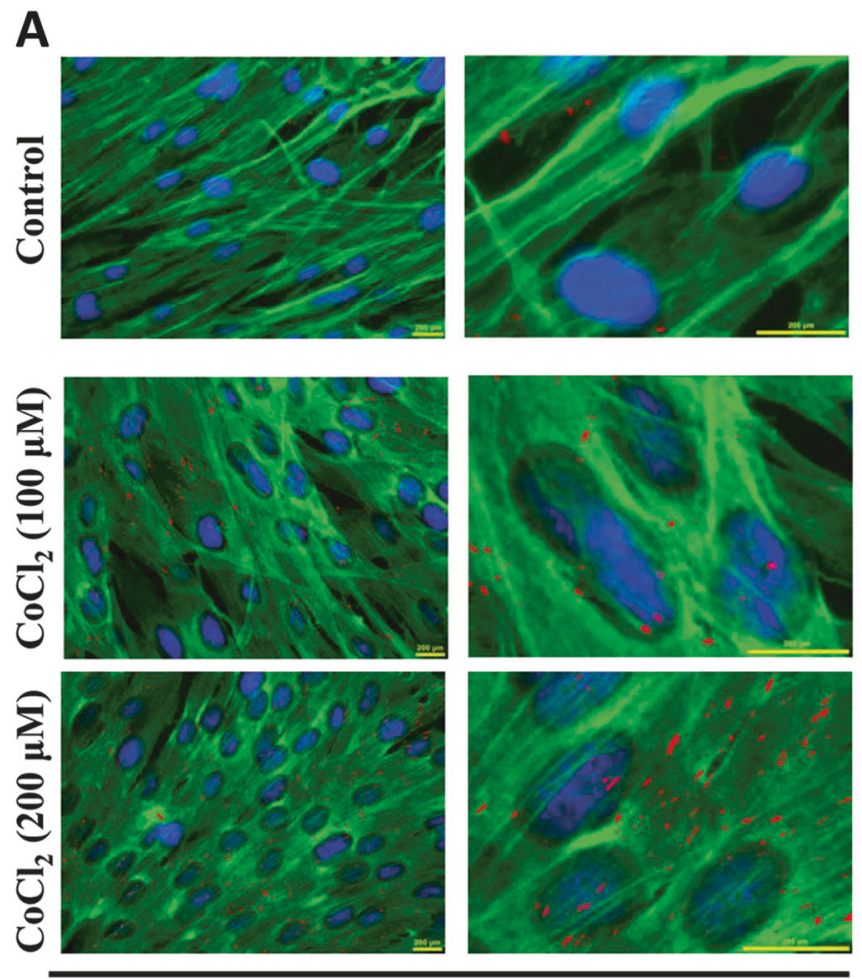

No AA treatment control

B

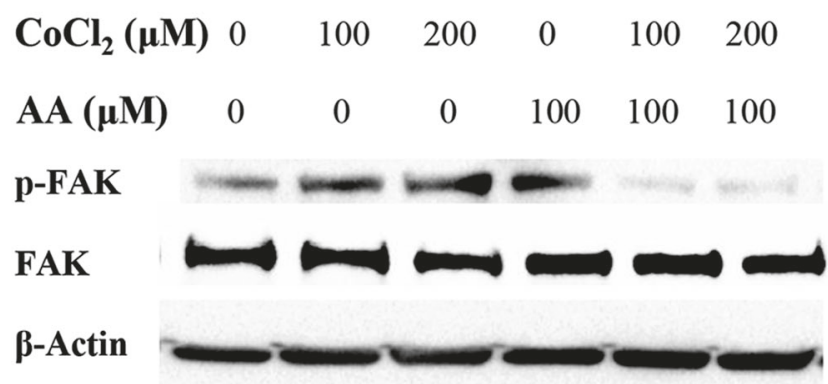

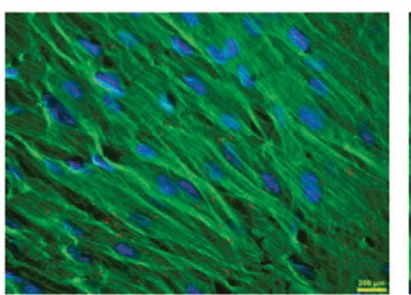
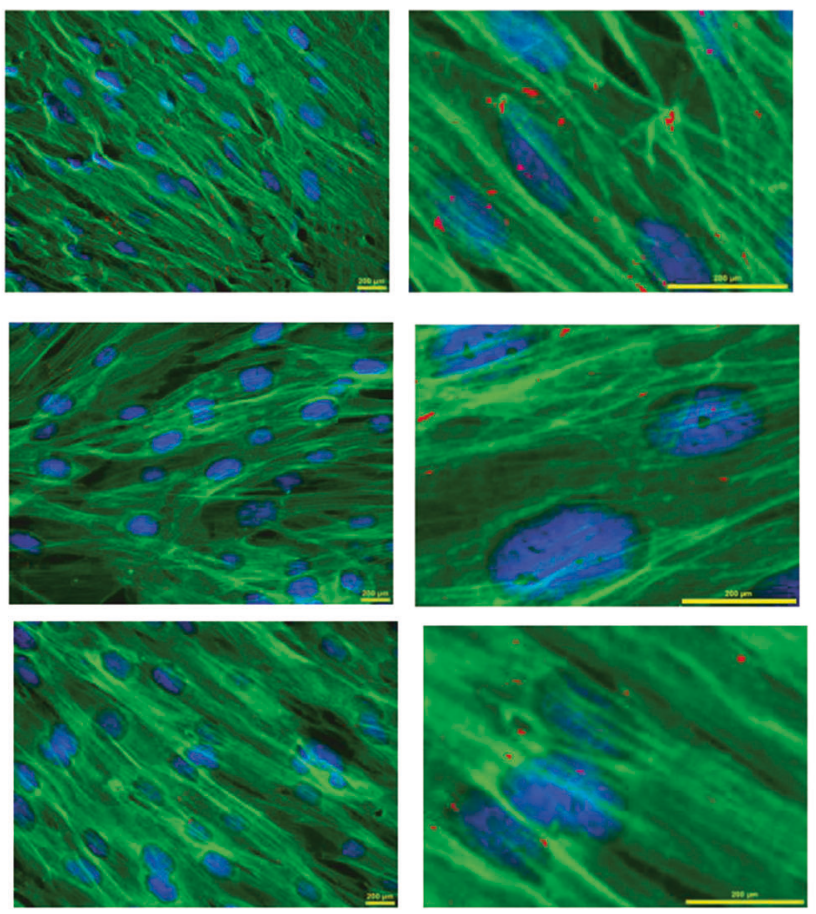

AA $(100 \mu M)$ for 24 hours

C Ratio of p-FAK / FAK levels
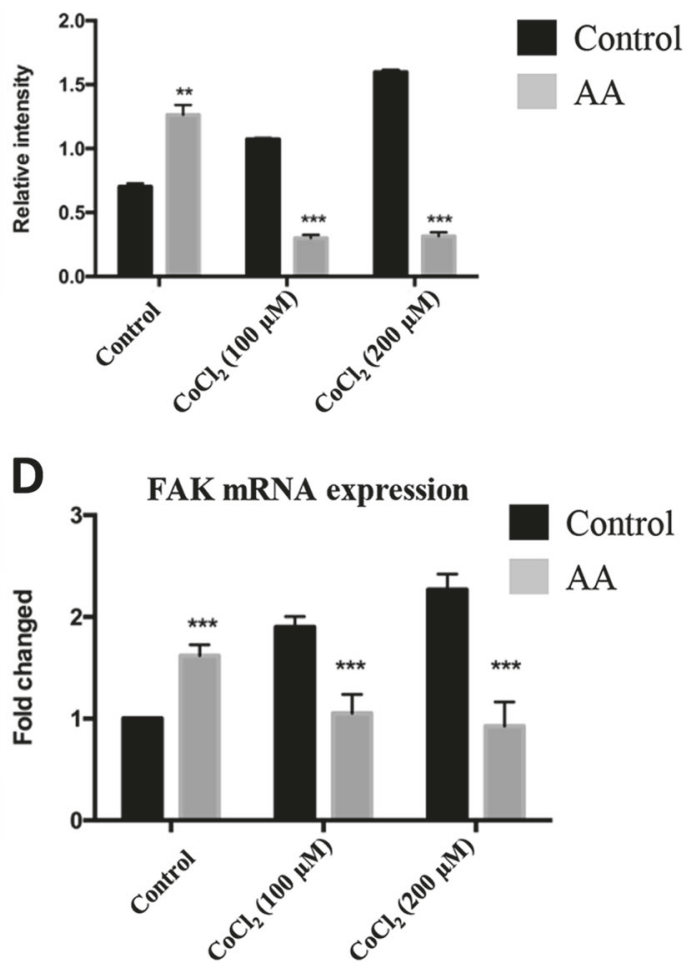

induced increase in ratio of p-FAK over total FAK expression, and d in FAK mRNA expression by qRT-PCR, as well as inhibition of this effect by AA $(* * * p<0.05 ; \mathrm{ns}=$ no significance $)$
Fig. 4 Effects of $\mathrm{CoCl}_{2}$ and AA treatment of human fibroblasts on phosphorylated focal adhesion kinase (p-FAK) expression. a Dual immunofluorescence (IF) staining for $\mathrm{F}$ actin (green) and p-FAK (red dots; blue $=$ DAPI). b, c Western blot showing $\mathrm{CoCl}_{2}$ (hypoxia)- 
These findings were reversed by treatment with ascorbic acid (Fig. 4b-d). In summary, the aggregate data suggest that hypoxic conditions that impact the TET3/5-hmC pathway also influence FAK in a manner to promote a phenotype seen in scar formation, and that epigenetic regulators such as ascorbic acid are capable of at least partially reversing such events.

\section{Discussion}

In this study, we have shown that (1) 5 -hmC is lost in human scar fibroblasts; (2) similar 5-hmC loss is experimentally inducible in cultured fibroblasts in vitro via $\mathrm{CoCl}_{2}$ induced hypoxia (confirmed by HIF-1 $\alpha$ elevation); (3) 5-hmC loss in hypoxic fibroblasts is associated with TET3 depletion and increased expression of FAK; and (4) these effects are at least partially diminished by ascorbic acid, thus providing a potential epigenetic mechanism of action for this agent.

Epigenetic modifications participate in multiple fibrosing disorders [24, 25], and DNA methylation plays an important role in maintaining cell types and function in scar formation. During the process of DNA demethylation, TET enzymes convert 5-mC to 5-hmC, utilizing $\mathrm{Fe}^{2+}$ and oxygen as co-factors and $\alpha$-ketoglutarate as a co-substrate. The TET family includes three different members: TET1, TET2 and TET3 [26]. It is known that mutations involving TET enzymes are important in cancer, and that TET expression is significantly reduced under conditions of tumor-related hypoxia [27, 28]. However, the effect of hypoxia on TET enzymes may be cell-type specific [27-30], and 5-hmC regulation during hypoxic conditions may relate to one or more of the members of the TET family, depending on the cellular processes that are affected. In our study, we provide evidence of 5-hmC loss in both conventional and hypertrophic scars, and demonstrate that this event may be experimentally replicated through induction of fibroblast hypoxia. In addition, the evidence suggests that unlike 5$\mathrm{hmC}$ loss in melanoma, which relates to TET2 deficiency [17], 5-hmC loss in scar formation appears to be more dependent on TET3. Thus, experimental induction of hypoxic conditions, as naturally occurs in scar formation, also resulted in 5-hmC loss that may be linked to effects on upstream TET3 $[4,30]$.

We noted that p-FAK was increased in association with hypoxia-induced 5-hmC loss in fibroblasts in vitro. FAK is a non-receptor protein tyrosine kinase which is capable of regulating cell adhesion, migration and growth [31]. It has been shown to be involved in integrin signaling and is overexpressed in numerous tumors [32, 33]. Previous studies have shown that mechanical loading after injury induces fibroblast-matrix interactions involving activated
FAK and resulting in skin fibrosis via the FAK-ERK-MCP1 pathway [34]. Moreover, suppression of FAK using RNA interference approaches has been shown to inhibit hypertrophic scar fibroblast proliferation [35]. Our study found that fibroblast p-FAK was increased when HIF-1 $\alpha$ increased, indicating that the phosphorylation of FAK may be activated by hypoxic conditions, in accordance with previous studies [36]. Although one prior study has demonstrated that TET1 knockdown activates FAK, the relationship between FAK and TET3 remains unclear [37]. Overall, while activating alterations in FAK are likely to contribute to fibrosis, they are unlikely to be exclusively the result of changes in the 5-hmC/TET3 axis.

Ascorbic acid has been reported to promote wound healing while inhibiting scar formation [38]. In embryonic stem cells, ascorbate promotes widespread DNA demethylation involving 1848 genes [39]. Ascorbate also has been shown to enhance TET-mediated generation of 5hmC [40, 41]. Accordingly, we examined this agent for its potential to rescue the changes observed as a consequence of fibroblast hypoxia in vitro. We found that ascorbic acid not only reversed the hypoxic status of fibroblasts, as indicated by HIF- $1 \alpha$, but also partially restored the loss of TET3 and 5-hmC. In order to address potential pleiotropic actions of ascorbic acid that extend beyond the HIF- $1 \alpha$ pathway, we also included a normoxia control group treated only with ascorbic acid. Interestingly, although HIF- $1 \alpha$ actually decreased slightly with treatment with ascorbic acid, our results indicate that the direct impact of ascorbic acid on 5-hmC, TET3, and FAK was likely to be negligible. Additionally, ascorbic acid suppressed the production of FAK [42]. It is notable that p-FAK also was elevated by ascorbic acid in fibroblasts under normoxic conditions, again suggesting a FAK effect independent of the 5-hmC/TET3 axis. Whether ascorbic acid directly mediated a decrease in HIF- $1 \alpha$ via the 5 -hmC/TET3 pathway remains unknown, although data exists indicating that ascorbic acid may directly decrease HIF- $1 \alpha$ activity in melanoma cells [43].

Limitations of this study include lack of data comprehensively evaluating the TET pathway at a protein level and utilization of TET knockdown approaches to better dissect the contributions of this pathway to the complex events inherent to scar formation. In addition, tissue concentrations of ascorbic acid must be considered inasmuch as absorption and urinary excretion of dietary supplements are altered with increasing doses, potentially limiting concentrations in the hypoxic tissues in which it is most needed. Thus, innovative modalities to achieve optimal tissue levels must also be considered, and combinatorial therapies that consider the epigenetic effects of agents like ascorbic acid in the context of other substances that thwart excessive scar formation require further translational study for future 
potential clinical applications. Our data have, however, established a model whereby hypoxia-related epigenetic alterations in the 5-hmC/TET axis may be further explored, and has provided potential insight into how modulators like ascorbic acid may be further evaluated for possible therapeutic interventions that will inhibit excessive scar formation at the level of the epigenome.

Scars can have devastating consequences on patients, both physiologically and psychologically. Conventional scar management includes surgical excision, compression therapy, intralesional corticosteroid injection, and laser therapy, among others [44, 45]. Since epigenetic modifications may have significant impact on fibroblast function, emerging therapies targeting DNA methylation, such as DNMT inhibitor treatment, are actively under investigation [46]. Now that mechanistic pathways are beginning to emerge, potentially simpler and more cost-effective approaches, such as ascorbic acid, should be seriously considered as a means of inhibiting traditional fibroblast activation and differentiation pathways that result in scar formation, thus providing a potential opportunity for more regenerative, stem cell-driven healing responses. In addition, oxygen level is likely to be important to wound healing before dysfunctional scar develops, and this point has potential impact on whether 5-hmC expression during earlier phases of the wound healing process may relate to differences in the rate and efficacy of the healing response. If so, then epigenetic regulators may also be of potential value in the important clinical situation of poorly healing skin ulcers.

Acknowledgements We are grateful for funding from the Harvard Stem Cell Institute that supported part of this initiative. Michael Wells provided valuable editorial assistance.

\section{Compliance with ethical standards}

Conflict of interest The authors declare that they have no conflict of interest.

Publisher's note: Springer Nature remains neutral with regard to jurisdictional claims in published maps and institutional affiliations.

\section{References}

1. Chodorowska G, Rogus-Skorupska D. Cutaneous wound healing. Ann Univ Mariae Curie Sklodowska Med. 2004;59:403-7.

2. Bando T, Yokoyama H, Nakamura H. Wound repair, remodeling, and regeneration. Dev Growth Differ. 2018;60:303-5.

3. Haverstock BD. Hypertrophic scars and keloids. Clin Podiatr Med Surg. 2001;18:147-59.

4. Zheng J, Song F, Lu SL, Wang WQ. Dynamic hypoxia in scar tissue during human hypertrophic scar progression. Dermatol Surg. 2014;40:511-8.
5. Sloan DF, Brown RD, Wells CH, Hilton JG. Tissue gases in human hypertrophic burn scars. Plast Reconstr Surg. 1978;61:431-6.

6. Iyer NV, Kotch LE, Agani F, Leung SW, Laughner E, Wenger $\mathrm{RH}$, et al. Cellular and developmental control of $\mathrm{O} 2$ homeostasis by hypoxia-inducible factor 1 alpha. Genes Dev. 1998;12:149-62.

7. Semenza GL. Regulation of mammalian $\mathrm{O} 2$ homeostasis by hypoxia-inducible factor 1. Annu Rev Cell Dev Biol. 1999;15:551-78.

8. Lokmic Z, Musyoka J, Hewitson TD, Darby IA. Hypoxia and hypoxia signaling in tissue repair and fibrosis. Int Rev Cell Mol Biol. 2012;296:139-85.

9. Scheid A, Wenger RH, Schaffer L, Camenisch I, Distler O, Ferenc A, et al. Physiologically low oxygen concentrations in fetal skin regulate hypoxia-inducible factor 1 and transforming growth factor-beta3. FASEB J. 2002;16:411-3.

10. Robinson CM, Watson CJ, Baugh JA. Epigenetics within the matrix: a neo-regulator of fibrotic disease. Epigenetics. 2012;7:987-93.

11. O'Reilly S. Epigenetics in fibrosis. Mol Asp Med. 2017;54:89102 .

12. Handy DE, Castro R, Loscalzo J. Epigenetic modifications: basic mechanisms and role in cardiovascular disease. Circulation. 2011;123:2145-56.

13. Zhang J, Chen S, Zhang D, Shi Z, Li H, Zhao T, et al. Tet3mediated DNA demethylation contributes to the direct conversion of fibroblast to functional neuron. Cell Rep. 2016;17:2326-39.

14. Neary R, Watson CJ, Baugh JA. Epigenetics and the overhealing wound: the role of DNA methylation in fibrosis. Fibrogenes Tissue Repair. 2015;8:18.

15. Zeisberg M, Kalluri R. Cellular mechanisms of tissue fibrosis. 1. Common and organ-specific mechanisms associated with tissue fibrosis. Am J Physiol Cell Physiol. 2013;304:C216-225.

16. Dowson C, O'Reilly S. DNA methylation in fibrosis. Eur J Cell Biol. 2016;95:323-30.

17. Lian CG, Xu Y, Ceol C, Wu F, Larson A, Dresser K, et al. Loss of 5-hydroxymethylcytosine is an epigenetic hallmark of melanoma. Cell. 2012;150:1135-46.

18. Ficz G, Gribben JG. Loss of 5-hydroxymethylcytosine in cancer: cause or consequence? Genomics. 2014;104:352-7.

19. Osawa T, Muramatsu M, Watanabe M, Shibuya M. Hypoxia and low-nutrition double stress induces aggressiveness in a murine model of melanoma. Cancer Sci. 2009;100:844-51.

20. Satish L, Lyons-Weiler J, Hebda PA, Wells A. Gene expression patterns in isolated keloid fibroblasts. Wound Repair Regen. 2006; $14: 463-70$

21. Huang C, Nie F, Qin Z, Li B, Zhao X. A snapshot of gene expression signatures generated using microarray datasets associated with excessive scarring. Am J Dermatopathol. 2013;35:6473.

22. De Felice B, Garbi C, Santoriello M, Santillo A, Wilson RR. Differential apoptosis markers in human keloids and hypertrophic scars fibroblasts. Mol Cell Biochem. 2009;327:191-201.

23. Wolff AC, Hammond ME, Schwartz JN, Dowsett M, McShane LM, Allison KH, et al. American Society of Clinical Oncology/ College of American Pathologists guideline recommendations for human epidermal growth factor receptor 2 testing in breast cancer. J Clin Oncol. 2007;25:118-45.

24. Stratton MS, McKinsey TA. Epigenetic regulation of cardiac fibrosis. J Mol Cell Cardiol. 2016;92:206-13.

25. Rabinovich EI, Kapetanaki MG, Steinfeld I, Gibson KF, Pandit $\mathrm{KV}, \mathrm{Yu} \mathrm{G}$, et al. Global methylation patterns in idiopathic pulmonary fibrosis. PLoS ONE 2012;7:e33770.

26. Kohli RM, Zhang Y. TET enzymes, TDG and the dynamics of DNA demethylation. Nature. 2013;502:472-9.

27. Hattori M, Yokoyama Y, Hattori T, Motegi S, Amano H, Hatada I, et al. Global DNA hypomethylation and hypoxia-induced 
expression of the ten eleven translocation (TET) family, TET1, in scleroderma fibroblasts. Exp Dermatol. 2015;24:841-6.

28. Mariani CJ, Vasanthakumar A, Madzo J, Yesilkanal A, Bhagat $\mathrm{T}$, Yu Y, et al. TET1-mediated hydroxymethylation facilitates hypoxic gene induction in neuroblastoma. Cell Rep. 2014;7: 1343-52.

29. Koivunen P, Laukka T. The TET enzymes. Cell Mol Life Sci. 2018;75:1339-48.

30. Laukka T, Mariani CJ, Ihantola T, Cao JZ, Hokkanen J, Kaelin WG Jr, et al. Fumarate and succinate regulate expression of hypoxia-inducible genes via TET enzymes. J Biol Chem. 2016;291:4256-65.

31. Parsons JT. Focal adhesion kinase: the first ten years. J Cell Sci. 2003;116(Pt 8):1409-16.

32. Mon NN, Ito S, Senga T, Hamaguchi M. FAK signaling in neoplastic disorders: a linkage between inflammation and cancer. Ann N Y Acad Sci. 2006;1086:199-212.

33. Hanks SK, Calalb MB, Harper MC, Patel SK. Focal adhesion protein-tyrosine kinase phosphorylated in response to cell attachment to fibronectin. Proc Natl Acad Sci USA. 1992;89:8487-91.

34. Wong VW, Rustad KC, Akaishi S, Sorkin M, Glotzbach JP, Januszyk M, et al. Focal adhesion kinase links mechanical force to skin fibrosis via inflammatory signaling. Nat Med. 2011;18:148-52.

35. Chen R, Zhang Z, Xue Z, Wang L, Fu M, Lu Y, et al. Focal adhesion kinase (FAK) siRNA inhibits human hypertrophic scar by suppressing integrin alpha, TGF-beta and alpha-SMA. Cell Biol Int. 2014;38:803-8.

36. Lee SH, Lee YJ, Song CH, Ahn YK, Han HJ. Role of FAK phosphorylation in hypoxia-induced hMSCS migration: involvement of VEGF as well as MAPKS and eNOS pathways. Am J Physiol Cell Physiol. 2010;298:C847-56.
37. Pei YF, Tao R, Li JF, Su LP, Yu BQ, Wu XY, et al. TET1 inhibits gastric cancer growth and metastasis by PTEN demethylation and re-expression. Oncotarget. 2016;7:31322-35.

38. Campos PM, Goncalves GM, Gaspar LR. In vitro antioxidant activity and in vivo efficacy of topical formulations containing vitamin $\mathrm{C}$ and its derivatives studied by non-invasive methods. Skin Res Technol. 2008;14:376-80.

39. Chung TL, Brena RM, Kolle G, Grimmond SM, Berman BP, Laird PW, et al. Vitamin C promotes widespread yet specific DNA demethylation of the epigenome in human embryonic stem cells. Stem Cells. 2010;28:1848-55.

40. Shenoy N, Bhagat T, Nieves E, Stenson M, Lawson J, Choudhary GS, et al. Upregulation of TET activity with ascorbic acid induces epigenetic modulation of lymphoma cells. Blood Cancer J. 2017;7:e587.

41. Minor EA, Court BL, Young JI, Wang G. Ascorbate induces teneleven translocation (Tet) methylcytosine dioxygenase-mediated generation of 5-hydroxymethylcytosine. J Biol Chem. 2013;288:13669-74.

42. Lagares D, Kapoor M. Targeting focal adhesion kinase in fibrotic diseases. BioDrugs. 2013;27:15-23.

43. Miles SL, Fischer AP, Joshi SJ, Niles RM. Ascorbic acid and ascorbate-2-phosphate decrease HIF activity and malignant properties of human melanoma cells. BMC Cancer. 2015;15:867.

44. Berman B, Maderal A, Raphael B. Keloids and hypertrophic scars: pathophysiology, classification, and treatment. Dermatol Surg. 2017;43(Suppl 1):S3-s18.

45. Gauglitz GG, Korting HC, Pavicic T, Ruzicka T, Jeschke MG. Hypertrophic scarring and keloids: pathomechanisms and current and emerging treatment strategies. Mol Med. 2011;17:113-25.

46. Russell SB, Russell JD, Trupin KM, Gayden AE, Opalenik SR, Nanney LB, et al. Epigenetically altered wound healing in keloid fibroblasts. J Invest Dermatol. 2010;130:2489-96. 